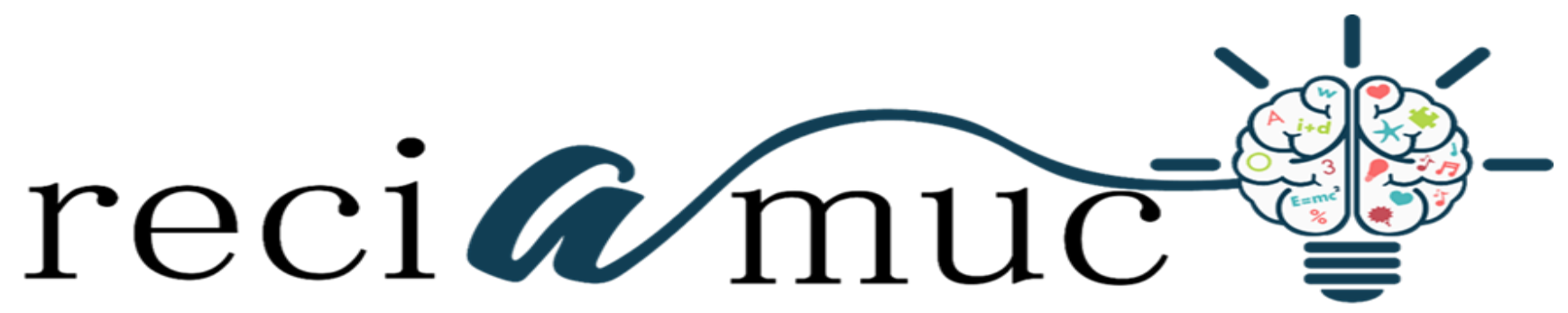

Revista cientifica de investigación actualización del mundo de las ciencias

Andrea Rosa Villón Chipre a; Johanna Katiuska Loja Vanegas ${ }^{\text {b; }}$ Nadia Lency

Pazmiño Peña ${ }^{c}$; Marcelo Antonio Alvarado Medrano ${ }^{d}$

Ventajas y desventajas del uso de catéter venoso central vía subclavia vs vía yugular interna

Advantages and disadvantages of the use of central venous cateter via subclavia vs internal yugular via

Revista Científica de Investigación actualización del mundo de las Ciencias. Vol. 3 núm., 2, abril, ISSN: 2588-0748, 2018, pp. 234-251

DOI: $10.26820 /$ reciamuc/3.(2).abril.2019.234-251

URL: $\underline{\text { http://reciamuc.com/index.php/RECIAMUC/article/view/335 }}$

Código UNESCO: 3205 Medicina Interna

Tipo de Investigación: Artículo de Revisión

(C) RECIAMUC; Editorial Saberes del Conocimiento, 2019

Recibido: 15/01/2019

Aceptado: 07/02/2019

Publicado: 01/04/2019

Correspondencia: andreavillonchipre@gmail.com
a. Médico; Investigador Independiente; Guayaquil, Ecuador; andreavillonchipre@gmail.com
b. Médico; Investigador Independiente; Guayaquil, Ecuador; medjlojav@ gmail.com
c. Médico; Investigador Independiente; Guayaquil, Ecuador; nlpazminoczs5@gmail.com
d. Médico; Investigador Independiente; Guayaquil, Ecuador; marceloantonio91@ hotmail.com 


\section{Ventajas y desventajas del uso de catéter venoso central vía subclavia vs vía yugular interna}

Vol. 3, núm. 2., (2019)

Andrea Rosa Villón Chipre; Johanna Katiuska Loja Vanegas; Nadia Lency Pazmiño Peña; Marcelo Antonio Alvarado Medrano

\section{RESUMEN}

Los dispositivos de acceso venoso central se utilizan en muchas ramas de la medicina donde se requiere acceso venoso a largo o corto plazo cuidado. Estas pautas revisan los tipos de dispositivos de acceso disponibles y hacen una serie de recomendaciones importantes. Se describen sus respectivas ventajas y desventajas en diversos entornos clínicos, cuidados del paciente antes de e inmediatamente después de la inserción se discute en el contexto de posibles complicaciones y cómo se evitan mejor. Hay una sección que trata el cuidado a largo plazo de los dispositivos en la vivienda. Se revisan las técnicas de inserción y extracción y se gestionan los problemas que tienen más probabilidades de ocurrir después de la inserción incluyendo infección, extravío y trombosis se discuten. Los cuidados del paciente con coagulopatías se abordan y hay una sección abordando problemas relacionados con el catéter.

Palabras claves: Catéter Venoso Central; Neumotórax; Embolia Aérea. 


\title{
Ventajas y desventajas del uso de catéter venoso central vía subclavia vs vía yugular interna
}

Vol. 3, núm. 2., (2019)

Andrea Rosa Villón Chipre; Johanna Katiuska Loja Vanegas; Nadia Lency Pazmiño Peña; Marcelo Antonio Alvarado Medrano

\begin{abstract}
Central venous access devices are used in many branches of medicine where long-term or shortterm venous access is required care. These guidelines review the types of access devices available and make a number of important recommendations. Their respective advantages and disadvantages are described in various clinical settings, patient care before and immediately after insertion is discussed in the context of possible complications and how they are best avoided. There is a section that deals with long-term care of devices in the home. Insertion and extraction techniques are reviewed and the problems that are most likely to occur after insertion are included, including infection, loss and thrombosis are discussed. Coagulopathic patient care is addressed and there is a section addressing catheter related problems.
\end{abstract}

Key words: Central Venous Catheter; Pneumothorax; Air Embolism. 


\section{Ventajas y desventajas del uso de catéter venoso central vía subclavia vs vía yugular interna}

Vol. 3, núm. 2., (2019)

Andrea Rosa Villón Chipre; Johanna Katiuska Loja Vanegas; Nadia Lency Pazmiño Peña;

Marcelo Antonio Alvarado Medrano

\section{Introducción.}

Existentes métodos de acceso de sangre para hemodiálisis se pueden dividir en dos amplias categorías: (1) subcutáneo, (2) transcutánea, que penetran en la piel de forma permanente. El más simple de acceso de sangre de la categoría subcutánea es un catéter de vena grande con entrada común semirrígido, que se retira después de una diálisis. Este método se utiliza principalmente para hemodiálisis aguda. Si el catéter se deja durante una diálisis, se puede considerar un híbrido de acceso subcutáneo y transcutánea. Para la hemodiálisis crónica un acceso subcutáneo se crea una anastomosis de la arteria y la vena directamente o a través de un injerto de bypass sintético. Las principales ventajas de un acceso subcutáneo son la longevidad y bajo riesgo de infección, complicaciones de acceso subcutáneas tales como flujo de salida y la entrada estenosis son en su mayoría relacionados con fístulas de injerto.

Las principales ventajas de los dispositivos transdérmicos son los siguientes: no hay necesidad de maduración, no hay necesidad de punción simple y no hay efectos perjudiciales de uso frecuente. Las principales desventajas son riesgo de infección, trombosis y estenosis de la vena resultante de tiempo de supervivencia limitada. No son considerados como una forma de acceso permanente para hemodiálisis crónica; sin embargo, los resultados están mejorando rápidamente en los últimos años. Además. en muchos pacientes que no son candidatos para la diálisis peritoneal y que hayan agotado todos los puntos de acceso de sangre subcutáneos, un acceso sanguíneo transcutánea sigue siendo el último recurso. 


\section{Ventajas y desventajas del uso de catéter venoso central vía subclavia vs vía yugular interna}

Vol. 3, núm. 2., (2019)

Andrea Rosa Villón Chipre; Johanna Katiuska Loja Vanegas; Nadia Lency Pazmiño Peña; Marcelo Antonio Alvarado Medrano

En esta breve reseña el enfoque será la cateterización de la vena yugular: sin embargo se mencionaran las ventajas y desventajas de la canulación de otras venas grandes: femoral y subclavia. Una extensa revisión de la literatura del acceso percutáneo para la hemodiálisis se ha publicado recientemente (Twardowski 1995).

\section{Metodología}

Para el desarrollo de este proceso investigativo, se plantea como metodología la encaminada hacia una orientación científica particular que se encuentra determinada por la necesidad de indagar en forma precisa y coherente una situación, en tal sentido (Davila, 2015) define la metodología "como aquellos pasos previos que son seleccionados por el investigador para lograr resultados favorables que le ayuden a plantear nuevas ideas". (p.66)

Lo citado por el autor, lleva a entender que el desarrollo de la acción investigativa busca simplemente coordinar acciones enmarcadas en una revisión bibliográfica con el fin de complementar ideas previas relacionadas con las ventajas y desventajas del uso de catéter venoso centralvia subclavia vs via yugular a través de una revisión de literatura, para así finalmente elaborar un cuerpo de consideraciones generales que ayuden a ampliar el interés propuesto.

\section{Tipo de Investigación}

Dentro de toda práctica investigativa, se precisan acciones de carácter metodológico mediante las cuales, se logra conocer y proyectar los eventos posibles que la determinan, así como las características que hacen del acto científico un proceso interactivo ajustado a una realidad 


\section{Ventajas y desventajas del uso de catéter venoso central vía subclavia vs vía yugular interna}

Vol. 3, núm. 2., (2019)

Andrea Rosa Villón Chipre; Johanna Katiuska Loja Vanegas; Nadia Lency Pazmiño Peña; Marcelo Antonio Alvarado Medrano

posible de ser interpretada. En este sentido, se puede decir, que la presente investigación corresponde al tipo documental, definido por Castro (2016), "se ocupa del estudio de problemas planteados a nivel teórico, la información requerida para abordarlos se encuentra básicamente en materiales impresos, audiovisuales y /o electrónicos". (p.41).

En consideración a esta definición, la orientación metodológica permitió la oportunidad de cumplir con una serie de actividades inherentes a la revisión y lectura de diversos documentos donde se encontraron ideas explicitas relacionadas con los tópicos encargados de identificar a cada característica insertada en el estudio. Por lo tanto, se realizaron continuas interpretaciones con el claro propósito de revisar aquellas apreciaciones o investigaciones propuestas por diferentes investigadores relacionadas con el tema de interés, para luego dar la respectiva argumentación a los planteamientos, en función a las necesidades encontradas en la indagación.

\section{Fuentes Documentales}

El análisis correspondiente a las características que predomina en el tema seleccionado, llevan a incluir diferentes fuentes documentales encargadas de darle el respectivo apoyo y en ese sentido cumplir con la valoración de los hechos a fin de generar nuevos criterios que sirven de referencia a otros procesos investigativos. Para (CASTRO, 2016) las fuentes documentales incorporadas en la investigación documental o bibliográfica, "representa la suma de materiales sistemáticos que son revisados en forma rigurosa y profunda para llegar a un análisis del fenómeno".(p.41). Por lo tanto, se procedió a cumplir con la realización de una lectura previa 


\section{Ventajas y desventajas del uso de catéter venoso central vía subclavia vs vía yugular interna}

Vol. 3, núm. 2., (2019)

Andrea Rosa Villón Chipre; Johanna Katiuska Loja Vanegas; Nadia Lency Pazmiño Peña; Marcelo Antonio Alvarado Medrano

determinada para encontrar aquellos aspectos estrechamente vinculados con el tema, con el fin de explicar mediante un desarrollo las respectivas apreciaciones generales de importancia.

\section{Técnicas para la Recolección de la Información}

La conducción de la investigación para ser realizada en función a las particularidades que determinan a los estudios documentales, tiene como fin el desarrollo de un conjunto de acciones encargadas de llevar a la selección de técnicas estrechamente vinculadas con las características del estudio. En tal sentido, (Bolívar, 2015), refiere, que es "una técnica particular para aportar ayuda a los procedimientos de selección de las ideas primarias y secundarias”. (p. 71).

Por ello, se procedió a la utilización del subrayado, resúmenes, fichaje, como parte básica para la revisión y selección de los documentos que presentan el contenido teórico. Es decir, que mediante la aplicación de estas técnicas se pudo llegar a recoger informaciones en cuanto a la revisión bibliográfica de los diversos elementos encargados de orientar el proceso de investigación. Tal como lo expresa, (Bolívar, 2015) "las técnicas documentales proporcionan las herramientas esenciales y determinantes para responder a los objetivos formulados y llegar a resultados efectivos" (p. 58). Es decir, para responder con eficiencia a las necesidades investigativas, se introdujeron como técnica de recolección el método inductivo, que hizo posible llevar a cabo una valoración de los hechos de forma particular para llegar a la explicación desde una visión general.

Asimismo, se emplearon las técnicas de análisis de información para la realización de la investigación que fue ejecutada bajo la dinámica de aplicar diversos elementos encargados de determinar el camino a recorrer por el estudio, según, (Bolívar, 2015) las técnicas de 


\section{Ventajas y desventajas del uso de catéter venoso central vía subclavia vs vía yugular interna}

Vol. 3, núm. 2., (2019)

Andrea Rosa Villón Chipre; Johanna Katiuska Loja Vanegas; Nadia Lency Pazmiño Peña; Marcelo Antonio Alvarado Medrano

procesamiento de datos en los estudios documentales "son las encargadas de ofrecer al investigador la visión o pasos que debe cumplir durante su ejercicio, cada una de ellas debe estar en correspondencia con el nivel a emplear" (p. 123). Esto indica, que para llevar a cabo el procesamiento de los datos obtenidos una vez aplicado las técnicas seleccionadas, tales como: fichas de resumen, textual, registros descriptivos entre otros, los mismos se deben ajustar al nivel que ha sido seleccionado.

\section{Resultados}

Perspectiva histórica

La ampliación de la cateterización venosa para hemodiálisis siguió su uso para otros fines. Es, por lo tanto, oportuno mencionar sus aplicaciones originales. La primera colocación de un catéter femoral para infusiones de soluciones hipertónicas se informó por Duffy en 1949 (Duffy 1949). Este método ganado popularidad en los últimos años como un procedimiento relativamente seguro, aunque varios autores informaron complicaciones tales como trombosis con embolia, tromboflebitis suppurat-ive, y la infección por el sitio inserción (Bonner 1951). Datos de autopsias en el informe de Bonner mostraron la formación de coágulos alrededor del propio catéter en cinco de 18 casos (Hoshal, Ause y Hoskins 2001). Estos casos representan probablemente la primera descripción documentada de lo que se conoce ahora como una manga de fibrina. La canulación de vasos femorales parahemodiálisis fue introducida por (Shaldon, Chiandussi y Briggs 2001) 


\section{Ventajas y desventajas del uso de catéter venoso central vía subclavia vs vía yugular interna}

Vol. 3, núm. 2., (2019)

Andrea Rosa Villón Chipre; Johanna Katiuska Loja Vanegas; Nadia Lency Pazmiño Peña; Marcelo Antonio Alvarado Medrano

La cateterización percutánea de la vena subclavia fue introducida por Aubaniac en 1952 y ha ganado popularidad después de que se demostró que era el sitio más conveniente de inserción del catéter de presión venosa central para el monitoreo de presión. Los autores recomendaron el método tan valioso y se asocia con baja tasa de complicaciones. Por el contrario, (Shapira y Stern 1997), basado en la experiencia obtenida por los demás ya sí mismos, recuperado el abandono de esta técnica. En su lugar, favorecieron la canalización a través de la vena yugular externa.

La canulación de la vena subclavia para hemodiálisis fue descrito por primera vez por (Erben, Kvasnicka y Bastecky 1969). Estos autores utilizaron dos catéteres de un solo lumen insertados en ambas venas subclavias ouno insertado en una vena subclavia y la otra en una vena femoral. La canalización de la vena yugular se ha practicado por generaciones de pediatras, pero en los adultos se introdujo el método en la década de 1960.

A principios de la década de 1980 se introdujeron los catéteres con dos lúmenes. Inicialmente catéteres rígidos de tetrafluoroetileno o poliuretano se han usado. El primer catéter de doble lumen fue desarrollado por Vas-Cath de Canadá y consistió en una canula arterial y una cánula venosa coaxialmente colocado. La cánula venosa fue sustituida por cada diálisis. El segundo catéter fue desarrollado por Quinton Instruments en Seattle WASHINGTON y consistió en diseño lumen septado (dual). Ambas compañías con diseños mejorados de sus catéteres son ampliamente utilizados para la terapia a corto plazo en la actualidad. 


\section{Ventajas y desventajas del uso de catéter venoso central vía subclavia vs vía yugular interna}

Vol. 3, núm. 2., (2019)

Andrea Rosa Villón Chipre; Johanna Katiuska Loja Vanegas; Nadia Lency Pazmiño Peña; Marcelo Antonio Alvarado Medrano

Los catéteres de caucho de silicona blanda para diálisis a largo plazo se utilizaron primero en lactantes y niños pequeños en la década de 1980.Unos años después del caucho de silicona de doble lumen, se usaron catéteres esposadas para hemodiálisis aguda y/o crónica.

\section{Catéteres sin manguito}

Un catéter insertado en una vena grande es muy útil como un acceso a la sangre para hemodiálisis aguda. Catéteres sin manguito semirrígidos se pueden insertar de forma rápida a través de la punción de la vena utilizando un método de inserción de Seldinger en un guía-alambre.

\section{Catéter femoral}

Lo más conveniente es la inserción en la vena femoral. El cateterismo femoral es rápido y sencillo. y en la mayoría de las instituciones que es un procedimiento de enfermería. Una punción arterial es la complicación más común debido a la posición variable de la vena femoral en relación a la arteria femoral. Ecografía Doppler de la zona inguinal es muy útil en casos difíciles. Los catéteres no pueden permanecer en su lugar durante mucho tiempo. Sin un manguito que restringe la migración de microorganismos, bacterias y / u hongos pueden alcanzar inevitablemente el torrente sanguíneo después de varios días. En pacientes ambulatorios el catéter no se debe dejar en la vena debido al riesgo de una punción en la vena de la pared. En pacientes no ambulatorios, el catéter puede dejarse en su lugar durante 4-5 días. 


\section{Ventajas y desventajas del uso de catéter venoso central vía subclavia vs vía yugular interna}

Vol. 3, núm. 2., (2019)

Andrea Rosa Villón Chipre; Johanna Katiuska Loja Vanegas; Nadia Lency Pazmiño Peña; Marcelo Antonio Alvarado Medrano

\section{Catéter de subclavia}

A diferencia de la vena femoral, la canulación de la vena subclavia fue pensado para el uso prolongado desde el inicio de este método en 1969. En comparación con el acceso femoral del sitio subclavia es más cómodo para los pacientes, que pueden deambular fácilmente, y el catéter se ha extendido la vida funcional. Por otro lado, la canalización subclavia de la vena no es un procedimiento de enfermería yse asocia con complicaciones de inserción más altos que el acceso femoral.

La estenosis de la vena subclavia, vena innominada. y vena cava superior se produce en hasta el 50\% de los casos debido a una canalización de la vena subclavia (Cimochowski, y otros 2000). La estenosis puede estar en silencio hasta que se crea una fístula arteriovenosa o injerto en el brazo ipsilateral. El más común es hinchazón del brazo ipsilateral con estenosis de la vena subclavia. La rigidez del catéter predisponela estenosis que se desarrolla en los lugares de torque máximo tal como entre la clavícula y la primera costilla particularmente en el lado derecho y en la vena braquiocefálica a cada lado. El catéter se frota contra la pared del vaso y daña la íntima. La lesión vascular conduce a la formación de trombos con la proliferación subendotelial posterior, fibrosis y la vena constricción. La estenosis es más propensa a desarrollarse en estos puntos.

\section{Catéter yugular}

Ambas venas yugulares externas e internas se pueden usar para la cateterización. Una canalización de la vena yugular no era popular como un acceso para hemodiálisis en la década de 1970 y principios de 1980, a pesar de los informes anteriores de que la cateterización yugular para 


\section{Ventajas y desventajas del uso de catéter venoso central vía subclavia vs vía yugular interna}

Vol. 3, núm. 2., (2019)

Andrea Rosa Villón Chipre; Johanna Katiuska Loja Vanegas; Nadia Lency Pazmiño Peña; Marcelo Antonio Alvarado Medrano

la monitorización vena central se asoció con menores tasas de complicaciones que estaba por debajo de la canulación de la vena subclavia. La canulación percutánea de la vena yugular es técnicamente más difícil debido a que los puntos de referencia anatómicos son menos prominentes que con la cateterización de la vena subclavia. Por otra parte, catéteres rectos rígidos que se extienden detrás de la oreja eran más difíciles para anclar y eran inconvenientes en pacientes ambulatorio en hemodiálisis que no le gustaban las restricciones de movimiento de la cabeza. Dos acontecimientos cambiaron la situación a finales de 1980 y principios de 1990: (1) varios documentos inequívocamente demostraron que la cateterización de la vena yugular es marcadamente menos probable de causar estenosis de la vena central que la cateterización de la vena subclavia (Cimochowski, y otros 2000). y (2) los nuevos catéteres con extensiones curvas preformada se hicieron disponibles: son mucho más cómoda y más fácil de asegurar (Mahurkar 1999).

El catéter se inserta más comúnmente en la vena yugular ya sea con una aproximación posterior (detrás del músculo esternocleidomastoideo) o con un enfoque 'anterior' a través del triángulo Sedillot (entre las cabezas esternal y clavicular del músculo esternocleidomastoideo) usando el método de Seldinger. Entonces la punta del catéter se hace avanzar a través de la vena braquiocefálica en la vena cava superior.

Catéteres semirrígidos no se deben insertar en la vena yugular izquierda debido al alto riesgo de trombosis y / o estenosis de la vena subclavia / braquiocefálico venoso debido a los caminos sinuosos. La inserción en la vena yugular derecha se asocia con la menor probabilidad de complicaciones mayores debido a una vía venosa casi en línea recta desde el sitio de inserción a 


\section{Ventajas y desventajas del uso de catéter venoso central vía subclavia vs vía yugular interna}

Vol. 3, núm. 2., (2019)

Andrea Rosa Villón Chipre; Johanna Katiuska Loja Vanegas; Nadia Lency Pazmiño Peña; Marcelo Antonio Alvarado Medrano

la aurícula derecha (Cimochowski, y otros 2000). El catéter puede dejarse en su lugar durante varias diálisis, incluso en pacientes ambulatorios.

\section{Catéteres esposados suaves}

Catéteres intravenosos esposados como acceso a sangre para hemodiálisis a largo plazo son de larga data de uso. En comparación con catéteres rígidos sin manguito, las principales ventajas de los catéteres blandos son un menor riesgo de trombosis, infección y estenosis de la vena, lo que resulta en tasas de supervivencia a largo plazo. La probabilidad de supervivencia de catéteres suaves, esposadas ha sido tan alta como $74 \%$ en 1 años en algunas series, valores similares a los conseguidos con fístula injerto subcutáneo (Gibson y Mosquera 2001).

La inserción quirúrgica en la derecha o la vena yugular interna izquierda a través del triángulo Sedillot es el método de preferido debido a la baja probabilidad de complicaciones tanto inmediato como a largo plazo. El catéter puede ser insertado a través del corte hacia abajo directa de la vena yugular. A diferencia de los catéteres rígidos, implantación de los catéteres blandos en las venas subclavia es menos popular debido a la aproximación más difícil. En muchos casos el método de desprendimiento debe ser aplicada debido a un corte hacia abajo directa no es posible. Además. las tasas de complicaciones son más bajos con el enfoque yugular. Los catéteres suaves rara vez se insertan en las venas femorales.

Los puntos de presión con catéter intravenoso recta insertan a través de la vena subclavia izquierda. Los puntos están situados en los lugares de torque máximo (flechas). (1) La entrada en la vena subclavia. (2) curva de la vena briachiocephalic delante del tronco braquiocefálico. (3) 


\section{Ventajas y desventajas del uso de catéter venoso central vía subclavia vs vía yugular interna}

Vol. 3, núm. 2., (2019)

Andrea Rosa Villón Chipre; Johanna Katiuska Loja Vanegas; Nadia Lency Pazmiño Peña; Marcelo Antonio Alvarado Medrano

vena cava superior. El daño de la íntima y la estenosis posterior puede desarrollarse en el espacio entre la clavícula y la primera costilla (punto).

\section{Complicaciones}

Trombosis. La formación de trombos es el principal impedimento a la longevidad del catéter. Un manguito de fibrina se forma probablemente en todos los catéteres, pero suele ser asintomatico. El retorno de la sangre deteriorada es generalmente el primer síntoma que indica que el manguito ha llegado a la punta del catéter. Si la instilación intraluminal no restaura la función del catéter, una infusión sistémica se necesita agente trombolítico. Si no hay contraindicación a los agentes trombolíticos, dosis desde 20000 a250 000 unidades de uroquinasapueden ser necesarias para restaurarla función del catéter. Un manguito de fibrina puede ser también eliminado por catéter de extracción. En este procedimiento una trampa avanzada de la vía femoral a través de la vena cava inferior y la aurícula derecha al catéter ocluido. El lazo se enrolla sobre el catéter apretado y tirado hacia abajo para quitar el manguito del catéter.

Un trombo peloto es un gran coagulo adherente a la punta del catéter, pero no a la pared del vaso. Un trombo mural es un gran coagulo adherente tanto al catéter y la pared del vaso causando la oclusión parcial de la embarcación. Tales trombos son más difíciles de disolver o tira que es un manguito fibrina y con frecuencia conducen a la extracción del catéter. Las embolias pulmonares asintomáticos deben ser extremadamente común con tantos acontecimientos trombóticos asociados con los catéteres. La mayoría de los coágulos se disuelven probablemente 


\section{Ventajas y desventajas del uso de catéter venoso central vía subclavia vs vía yugular interna}

Vol. 3, núm. 2., (2019)

Andrea Rosa Villón Chipre; Johanna Katiuska Loja Vanegas; Nadia Lency Pazmiño Peña; Marcelo Antonio Alvarado Medrano

de manera eficiente en el pulmón sin manifestación clínica debido a informes sobre embolias pulmonares son raros.

Prevención de una trombosis relacionada con el catéter se basa en el uso de los materiales trombogénicos, la mejor ruta de inserción, y la prevención de estados hipercoagulables. Una ruta yugular, particularmente en el lado derecho es ventajoso como tener casi una línea recta y por lo tanto minimiza trauma a la íntima. Infecciones deben ser tratados vigorosamente porque son asociadas con estado de hipercoagulabilidad. La anticoagulación en el 'catéter de diálisis' debe ser más vigoroso que en la 'fístula de diálisis' y el contacto de la sangre con la membrana de diálisis activa el sistema de coagulación. A diferencia de 'fístula de diálisis' el 'catéter de diálisis' requiere la continuación de la anticoagulación eficiente hasta el final de la diálisis para evitar la deposición de coágulos en el catéter en ese momento. Por otra parte, no hay ningún riesgo de hemorragia en los lugares de punción después de la diálisis. Si el paciente tiene una tendencia a la hemorragia, solamente un citrato debe ser utilizado para anticoagulación. Los anticoagulantes orales parecen valiosos en la prevención de una trombosis relacionada con el catéter.

Infecciones. Estos incluyen sitio de salida / túnel infección, sepsis, y tromboflebitis séptica. De manera similar a los catéteres sin manguito, los microorganismos pueden ser introducidos transluminalmente o periluminalmente; la semilla ciónhematógena del catéter desde un lugar de la infección a distancia también es posible. El manguito constituye una barrera significativa para la penetración bacteriana periluminal, y las tasas de infección con catéteres con balón son marcadamente más baja que con los catéteres sin manguito. infección de salida es por lo generaldia gnosed por la presencia de dolor. eritema y purulenta secreción del sitio de salida. 


\section{Ventajas y desventajas del uso de catéter venoso central vía subclavia vs vía yugular interna}

Vol. 3, núm. 2., (2019)

Andrea Rosa Villón Chipre; Johanna Katiuska Loja Vanegas; Nadia Lency Pazmiño Peña; Marcelo Antonio Alvarado Medrano

Un diagnóstico diferencial de cualquier reacción febril con o sin sangre elevado recuento de glóbulos blancos en un paciente con un catéter intravenoso incluye sepsis relacionada con el catéter. El cuadro clínico puede variar desde febrícula oligosintomático a un shock séptico grave, potencialmente mortal. Obviamente, no todas las reacciones febriles en pacientes con catéteres permanentes indican sepsis relacionada con el catéter. Un diagnóstico diferencial apropiado es importante para evitar la pérdida innecesaria de un catéter "inocente". Por otra parte, un retraso en la retirada del catéter responsable de la sepsis puede llevar a un shock séptico con graves consecuencias. Un enfoque meticuloso al diagnóstico y tratamiento de una reacción febril en un paciente con un catéter intravenoso ha sido descrito por (Capdevila y Segarra 2003).

La combinación de la sepsis y trombosis puede conllevar al desarrollo de tromboflebitis supurativa. Las características clínicas que sugieren este diagnóstico incluyen:

- Bacteriemia persistente después de la retirada del catéter a pesar de la terapia antibiótica apropiada.

- A falta de pruebas de la endocarditis.

- Trombosis venosa central feblograficamente demostrado.

- La respuesta clínica y bacteriológica con la institución de la terapia de heparina.

\section{Conclusiones}

Los catéteres intravenosos para la hemodiálisis son muy útiles como accesos de sangre. Catéteres sin manguito semirrígidos se pueden insertar de forma rápida a través de una punción de la vena utilizando el método de inserción de Seldinger en un guía-alambre. Lo más conveniente es 


\section{Ventajas y desventajas del uso de catéter venoso central vía subclavia vs vía yugular interna}

Vol. 3, núm. 2., (2019)

Andrea Rosa Villón Chipre; Johanna Katiuska Loja Vanegas; Nadia Lency Pazmiño Peña; Marcelo Antonio Alvarado Medrano

la inserción en la vena femoral. La cateterización de la yugular y la vena subclavia preferiblemente deben reservarse para catéteres suaves esposadas debido a la alta probabilidad de infección. trombosis venosa y la estenosis con rigidez de los catéteres sin manguito. La canulación de la vena yugular derecha se asocia con las tasas de complicaciones más bajas. Las tasas de supervivencia de catéteres blandos son comparables a los de las fístulas injerto en algunas series.

\section{Bibliografía}

Bolívar, J. Investigación Documental. México. Pax, 2015.

Bonner, CD. « Experience with plastic tubing in prolonged intravenous therapy. » N England J Med, 1951: 97-98.

Capdevila, JA, y A. Segarra. «Successful treatmemt of haemodialysis catheter-related sepsis without catheter removal. » Planes AM, 2003: 231-234.

Castro, J. Técnicas Documentales. México. Limusa, 2016.

Cimochowski, GE, E Worley, WE Rutherford, J Sartain, J Blondin, y H. Harter. «Superiority of the internal jugular over the subclavian access for temporary dialysis.» Nephron, 2000: 154-161.

Davila, A. Concepto de terminos cientificos. Caracas: Oasis, 2015.

Duffy, B Jr. «Clinical use of polyethylene tubing for intravenous therapy. Report on 72 cases. .» Ann Surg, 1949: 929-936.

Erben, J, J Kvasnicka, y J. Bastecky. «Experience with routine usc of subclavian vein cannulation in haemodialvsis.» Eur Dial Transplam Assoc., 1969: 59-64. 


\section{Ventajas y desventajas del uso de catéter venoso central vía subclavia vs vía}

yugular interna

Vol. 3, núm. 2., (2019)

Andrea Rosa Villón Chipre; Johanna Katiuska Loja Vanegas; Nadia Lency Pazmiño Peña; Marcelo Antonio Alvarado Medrano

Gibson, SP, y D. Mosquera. «Five years experience with the Quinton Permcath for vascular access.» Vephrol Dial Trcmsplcmt , 2001: 269-274.

Hoshal, VL Jr, RG Ause, y PA. Hoskins. «Fibrin sleeve formation indwelling subclavian central venous catheters.» Arch Surg, 2001: 373-375.

Mahurkar, SD. «Memon-induced curved extensions for Mahurkar catheters. .» Dial Trausplalll, 1999.

Shaldon, S, L Chiandussi, y B. Briggs. «Haemodialysis by percutaneous catheterisation of the femoral artery and vein with regional heparinisation. .» 2001: 857-859.

Shapira, M, y WZ. Stern. «Hazards of subclavian vein cannulation for central venous pressure monitoring. .» JAMA, 1997: 111-113.

Twardowski, Z. «Percutaneous blood access for hemodialysis.» Semin Dia, 1995: 175-186.

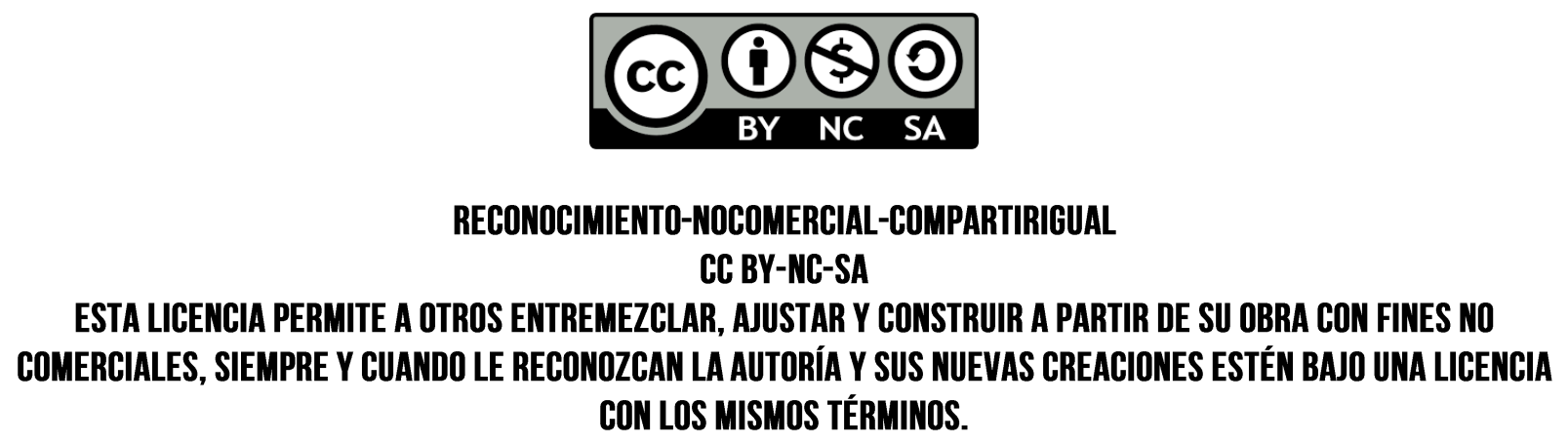

